\title{
Anosmia as a prominent symptom of COVID-19 infection*
}

\author{
Firouzeh Heidari', Ebrahim Karimi', Mohammadreza Firouzifar', Parnian $\quad$ Rhinology 58: 3, 302- -303, 2020 \\ Khamushian2, Reza Ansari', Mojtaba Mohammadi Ardehali', Farrokh Heidari' https://doi.org/10.4193//hhin20.140 \\ 'Otorhinolaryngology Research Center, Tehran University of Medical Sciences, Tehran, Iran \\ *Received for publication: \\ ${ }^{2}$ Internal Medicine Research Center, Tehran University of Medical Sciences, Tehran, Iran \\ April 9, 2020 \\ Accepted: April 14, 2020
}

According to $\mathrm{WHO}$ recommendations, everyone must protect themselves against Coronavirus disease 2019 (COVID-19), which will also protect others. Due to the lack of current effective treatment and vaccine for COVID-19, screening, rapid diagnosis and isolation of the patients are essential ${ }^{(1,2)}$.

Therefore, identifying the early symptoms of COVID-19 is of particular importance and is a health system priority. Early studies from COVID-19 outbreak in China have illustrated several non-specific signs and symptoms in infected patients, including fever, dry cough, dyspnea, myalgia, fatigue, lymphopenia, and radiographic evidence of pneumonia ${ }^{(3,4)}$. Recently, a probability of association between COVID-19 and altered olfactory function has been reported in South Korea, Iran, Italy, France, UK and the United States ${ }^{(5-8)}$. However, to our knowledge, the definite association between COVID-19 and anosmia has not been published.

Here, we conducted a consecutive case series study. From March 1, 2020, twenty-three cases with a chief complaint of recent isolated anosmia and confirmed COVID-19 infection were enrolled. Patients with symptoms or past medical histories of allergy, sinonasal disease (nasal congestion, rhinorrhea, sneeze, and facial pain or fullness), sinonasal surgery, neurological disease (multiple sclerosis, brain tumor, Parkinson, dementia, and seizure), head trauma, HIV infection, head and neck radiotherapy, and recent hospitalization in the last month were excluded. A confirmed case with COVID-19 was defined as a positive result of real-time reverse-transcriptase polymerase-chain-reaction (RT-PCR) assay for nasal and pharyngeal swab specimens and only the confirmed cases were included in the analysis.

Mean age of patients was 37.4 years old, including 8 men and 15 women. Sixteen cases had only anosmia, 3 cases reported low grade fever, and 4 cases reported Mild myalgia and fatigue. The fever, myalgia and fatigue had improved in the first few days, but the anosmia remained. Nineteen patients (83\%) reported anosmia as their first symptoms.

Olfactory and taste dysfunction can occur following the wide range of upper respiratory tract infections which is named postviral anosmia. However, the exact underlying pathogenesis is not well understood ${ }^{(9)}$.

Post-viral anosmia is more common in women and it has favorable prognosis unlike other subgroups of anosmia ${ }^{(5,7)}$. In this study, two-thirds of patients were female and about $75 \%$ $(17 / 23)$ of patients reported significant improvement in olfactory function after 2 weeks of follow up.

Our observation shows that anosmia could be the only clinical presentation of COVID-19 without any other significant symptoms. Therefore, we suggests that new onset anosmia should be considered in routine work-up and screening for COVID-19 infection. Also, self-isolation could be recommended for individuals with anosmia in COVID-19 outbreak. However, determining of the incidence, etiology, prognosis, and therapeutic strategy in patients with olfactory dysfunction require more investigations.

Key words: anosmia, olfactory dysfunction, loss of sense of smell, COVID-19, corona virus, 2019-nCoV

\section{References}

1. Organization WH. Coronavirus Disease 2019 (COVID-19) Situation Reports.

2. Wang C, Horby PW, Hayden FG, Gao GF. A novel coronavirus outbreak of global health concern. Lancet. 2020;395(10223):470-3

3. Wang D, Hu B, Hu C, Zhu F, Liu X, Zhang $J$, et al. Clinical characteristics of 138 hos- pitalized patients with 2019 novel coronavirus-infected pneumonia in Wuhan China. JAMA. 2020 Feb 7. doi: 10.1001/ jama.2020.1585.

4. Wu F, Zhao S, Yu B, Chen Y-M, Wang W, Song Z-G, et al. A new coronavirus associated with human respiratory disease in China. Nature. 2020;579(7798):265-9.
5. Bagheri SHR, Asghari AM, Farhadi M Shamshiri AR, Kabir A, Kamrava SK, et al. Coincidence of COVID-19 epidemic and olfactory dysfunction outbreak. medRxiv 2020.03.23.20041889; doi: https://doi.org/1 0.1101/2020.03.23.20041889

6. Giacomelli A, Pezzati L, Conti F, Bernacchia D, Siano M, Oreni L, et al. Self-reported 
olfactory and taste disorders in SARS-CoV-2 patients: a cross-sectional study. Clinical Infectious Diseases, ciaa330, https://doi. org/10.1093/cid/ciaa330

7. Hopkins C, Kumar N. Loss of sense of smell as marker of COVID-19 infection. Retrieved from www.entuk.org/sites/default/files/ files/Loss $\% 20$ of $\% 20$ sense $\% 20$ of $\% 20$ smell\%20as\%20marker\%20of\%20COVID.pdf 2020

8. lacobucci G. Sixty seconds on... anosmia. BMJ. 2020 Mar 24;368:m1202.

9. Brann D, Tsukahara T, Weinreb C, Logan DW, Datta SR. Non-neural expression of SARS-CoV-2 entry genes in the olfactory epithelium suggests mechanisms underlying anosmia in COVID-19 patients. bioRxiv 2020.03.25.009084; doi: https://doi. org/10.1101/2020.03.25.009084.

Farrokh Heidari, MD-MPH

Otorhinolaryngology Research

Center

Amir Alam Hospital

North Sadi Ave

Tehran 1145765111

Iran

Tel: 0982166760269

E-mail: farrokh.heidari@yahoo.com 